\title{
Thermo-optical Arrays of Flexible Nanoscale Nanomembranes Freely Suspended over Microfabricated Cavities as IR Microimagers
}

\author{
Chaoyang Jiang ${ }^{\dagger}$, Michael E. McConney ${ }^{\dagger}$, Srikanth Singamaneni ${ }^{\dagger}$, Emily Merrick ${ }^{\dagger}$, \\ Yuchuan Chen ${ }^{\ddagger}$, Jing Zhao ${ }^{\ddagger}$, Lei Zhang ${ }^{\ddagger}$, and Vladimir V. Tsukruk ${ }^{{ }^{*}}$ \\ Department of Materials Science and Engineering, Iowa State University, Ames, IA \\ 50011, and Agiltron Incorporated, Woburn, MA 01801
}

\section{Support Information}

Poly(allyl amine) (PAH) and poly(sulfonated styrene) (PSS) were purchased from Aldrich and used as received. Gold nanoparticles were synthesized according to the know procedure. ${ }^{1,2}$ The freely suspended nanomembranes were fabricated with SA-LbL assembly as reported elsewhere. Error! Bookmark not defined.b, Error! Bookmark not defined. In this study, we assembled and used membranes that were composed of a monolayer of gold nanoparticles encapsulated between 11 polymer bilayers from each side $(\mathrm{PAH} / \mathrm{PSS})_{11} \mathrm{PAH} / \mathrm{Au} /(\mathrm{PAH} / \mathrm{PSS})_{11} \mathrm{PAH} \quad$ or, briefly, $11 \mathrm{G} 11$ with the internal microstructure presented in Fig. 1a. Such multilayered nanocomposite membranes were applied to seal the microcavities of the micromachined substrate. The thermal expansion and contraction of the air inside the microcavity result in the positive/negative pressure differential and corresponding deflection of the flexible membrane which caps the silicon microcavity (Fig. 1b).

The surface morphology and membrane thickness were examined with a Dimension 3000 atomic force microscope (AFM) (Digital Instruments). The microcavity arrays were fabricated from silicon wafers. Cavities can be designed and fabricated in various shapes, sizes and depths, but in this study we focused on cylindrically-shaped cavities with 80 $\mu \mathrm{m}$ diameters and $70 \mu \mathrm{m}$ depths. The half-inch $64 \times 64$ array of microcavities was

\footnotetext{
* To whom correspondence should be addressed. E-mail: vladimir@iastate.edu

${ }^{\dagger}$ Iowa State University

${ }^{\ddagger}$ Agiltron Incorporated
} 
fabricated by etching the cylindrical holes using the DRIE processing. ${ }^{3,4} \mathrm{SiO}_{2}$ was used as the etching stop layer. SEM micrographs of the silicon substrate with microcavity arrays were collected with JSM-6060LV.

The thermal sensitivity of the microcavity arrays was measured under ambient conditions with a custom-built interferometeric set-up as described earlier. ${ }^{5}$ During the measurement, the relative humidity (RH) in the laboratory was monitored and we found that RH in the range of 20-30\% did not affect freely suspended SA-LbL nanomembranes behavior significantly contrary to conventional LbL films as was discussed

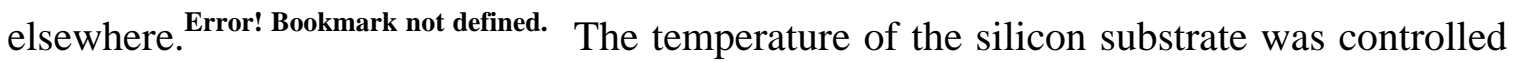
with a Peltier thermoelectric element (Melcor Corp.) coupled to an IDT-5948 precision temperature controller (ILX Lightwave Corp.). The temperature was measured with a calibrated thermistor with an accuracy of $\pm 0.01^{\circ} \mathrm{C}$ and a temperature stability of $\pm 0.05^{\circ} \mathrm{C}$. The interferometer patterns were recorded with a CCD camera and analyzed by using

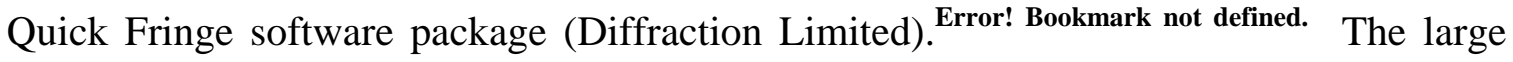
deflections of the nanomembranes were also observed with optical microscopy. Near-IR (NIR) laser (785 nm, $150 \mathrm{~mW}, 0.5 \mathrm{~mm}$ beam diameter) was used to illuminate selected areas of the array.

Finite element analysis (FEA) of the thermal bulging exploited a commercial FEA package, COMSOL Mutilphysics 3.2. ${ }^{6}$ A FEA model of a cylindrical cavity containing air (thermal expansion coefficient, $\alpha=9 \times 10^{-3} / K, v \approx 0.5$ ) sealed with the flexible freely suspended nanomembrane (elastic modulus $6 \mathrm{GPa}$ as estimated in our previous studies) was meshed into 35,000 elements and the energy differential was constructed from the strain matrix computed from the thermal stresses caused by to the expansion of the air within the microcavity.

In fact, the polymer membrane 11G11 uniformly covered the microfabricated array as seen with optical microscopy which shows a perfect array of circular reflections (Fig. S1a). The membrane was planar state at room temperature with bright central spots caused by strong reflection from the bottom of the microcavities (Fig. S1b). The edge of 
the nanomembrane can be easily identified with optical microscopy by dramatic change in optical reflectance (Fig. S1b). From the AFM imaging of the edge region the topography and the thickness of 11G11 nanomembrane were obtained (Fig. S1c). The 11G11 film had a total thickness of $85 \mathrm{~nm}$ as measured from the section analysis. The surface of 11G11 membrane was very smooth with the microroughness of $10 \mathrm{~nm}$ within $2 \times 2 \mu \mathrm{m}$ caused by the presence of the gold nanoparticles with diameter $13 \mathrm{~nm}$ inside of the nanomembrane (Fig. S1c, d).
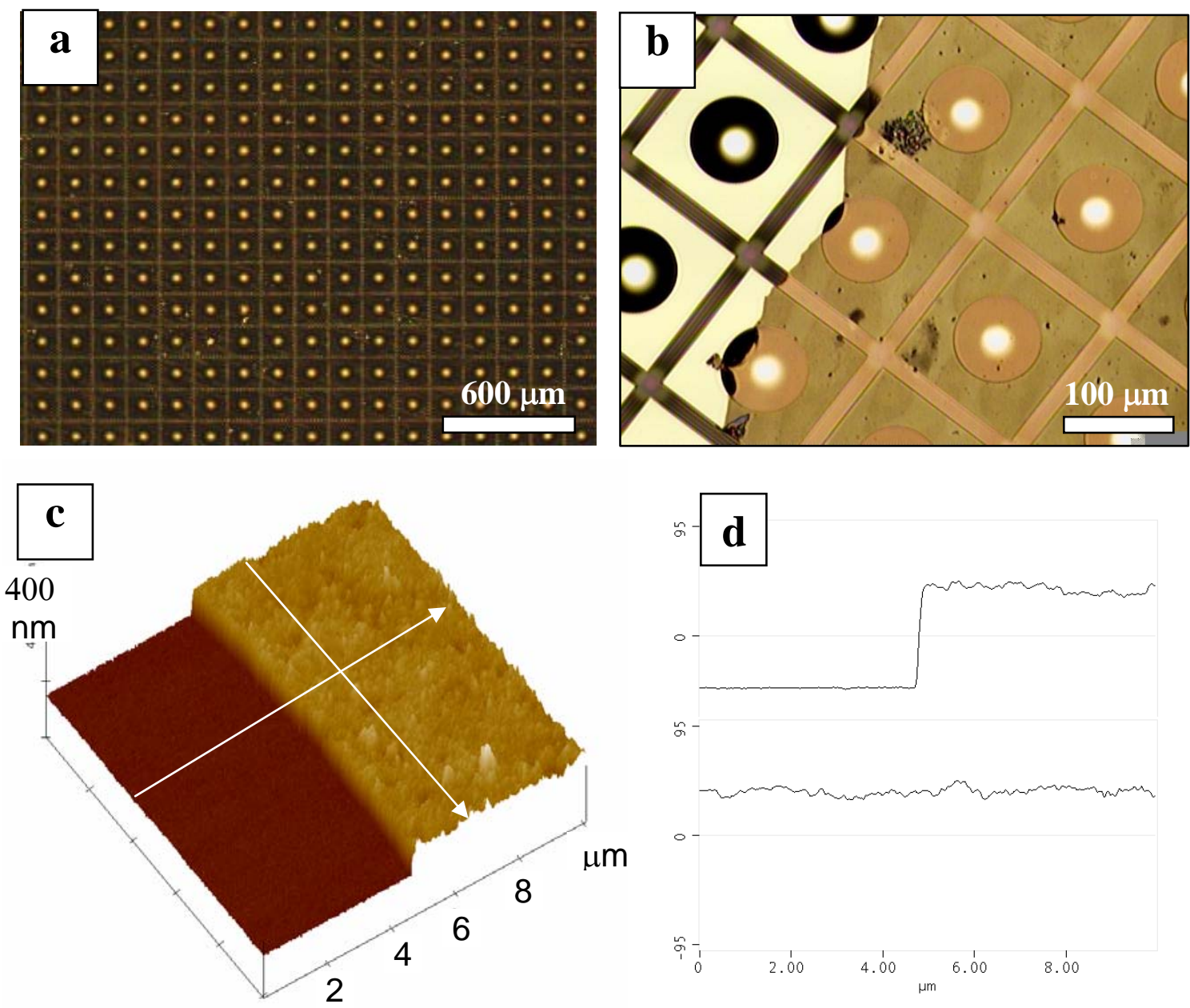

Figure S1. (a) Optical micrograph of microcavity array at $27^{\circ} \mathrm{C}$. (b) Optical image of the nanomembrane partially covering the microcavity array, left top corner shows a bare chip surface with much higher reflectance. (c) 3D AFM image of the edge of the 
nanomembrane on silicon substrate with section analysis (d): across the edge (top) and along the edge (bottom).

(1) Grabar, K. C.; Freeman, R. G.; Hommer, M. B.; Natan, M. J. Anal. Chem. 1995, 67, 735.

(2) Jiang, C.; Markutsya, S.; Tsukruk, V. V. Langmuir 2004, 20, 882.

(3) Yan, D.; Cheng, J.; Apsel, A. Sens. Actuat. A 2004, 115, 60.

(4) Yan, D.; Apsel, A.; Lal, A. Smart Mater. Struct. 2005, 14, 775.

(5) Markutsya, S.; Jiang, C.; Pikus, Y.; Tsukruk, V. V. Adv. Funct. Mater. 2005, 15, 771.

(6) For details of FEA package see http://www.comsol.com/ 